\title{
Força de trabalho de Profissionais de Educação Física na Atenção Primária à Saúde
}

\author{
Workforce of Physical Education Professionals in Primary Health Care
}

\section{AUTORES \\ Debora Bernardo da Silva ${ }^{1}$ (ic \\ Taciana Rocha dos Santos Sixel ${ }^{2}$ (D) \\ Arthur de Almeida Medeiros ${ }^{2}$ (DD \\ Ana Carolina Basso Schmitt ${ }^{3}$ (D) \\ 1 Universidade de São Paulo, Faculdade de Saúde Pública, Departamento de Epidemiologia, São Paulo, São Paulo, Brasil. \\ 2 Universidade de São Paulo, Faculdade de \\ Medicina, Departamento de Fisioterapia, Fonoaudiologia e Terapia Ocupacional, São Paulo, São Paulo, Brasil. \\ 3 Universidade Federal do Mato Grosso do Sul, Instituto Integrado de Saúde, Campo Grande, Mato Grosso do Sul, Brasil.}

\section{CONTATO}

Debora Bernardo da Silva

deborabernardo@usp.br

Rua Doutor Arnaldo, n. 715. São Paulo, São

Paulo, Brasil.

CEP: 01246-904.

DOI

$10.12820 /$ rbafs. $27 \mathrm{e} 0240$

\section{(cc) BY}

Este trabalho está licenciado com uma Licença Creative Commons - Atribuicão 4.0 Internacional.

\begin{abstract}
RESUMO
O objetivo deste estudo foi analisar a distribuição espaço temporal de Profissionais de Educação Física na Atenção Primária à Saúde do Sistema Único de Saúde nos Estados brasileiros. Este estudo possui característica ecológica de série temporal, em que foram utilizados dados secundários do Cadastro Nacional de Estabelecimentos de Saúde de 2008 a 2020 para identificar a força de trabalho desta área profissional. Foram construídos mapas e gráficos para a análise espacial da força de trabalho, número de Profissionais de Educação Física por 10.000 habitantes, por Estados e regiões brasileiras. As análises de regressão foram realizadas no Joinpoint Regression Program versão 4.7.0.0, considerando o intervalo de confiança de 95\%. Em 2008 no Brasil havia 321 Profissionais de Educação Física na atenção primária e 139 em 2020, representando uma diminuição de 56\% para a quantidade de profissionais vinculados à Atenção Primária à Saúde. Observou-se o crescimento da densidade deste profissional na maioria dos Estados no primeiro momento e o decréscimo em seguida. Destaca-se o Estado de Espírito Santo, com a maior densidade de profissionais em 2008 e Pernambuco que atingiu em 2012 o maior pico entre os Estados. A tendência temporal variou entre os Estados brasileiros, Goiás foi o único que apresentou aumento no período de 2008 a 2020, já na região Sudeste todos os Estados demonstraram decréscimo. $\mathrm{O}$ incentivo da prática de atividade física supervisionada e orientada é importante para auxiliar no aumento da força de trabalho do Profissional de Educação Física na Atenção Primária à Saúde, como também aumentar o nível de atividade física da população, contribuindo para adquirir benefícios de saúde oriundos da prática regular de atividade física.
\end{abstract}

Palavras-chave: Atividade física; Sistema Único de Saúde; Epidemiologia; Educação física; Força de trabalho.

\section{ABSTRACT}

The aim of this study was to analyze the temporal distribution of Physical Education Professionals in Primary Health Care in the Unified Health System in Brazilian States. This study has an ecological feature of a time series, in which secondary data from the National Registry of Health Facilities from 2008 to 2020 were used to identify the workforce of this professional area. Maps and graphs were built for the spatial analysis of the workforce, number of Physical Education Professionals per 10,000 inhabitants, by Brazilian states and regions. Regression analyzes were performed using the Joinpoint Regression Program version 4.7.0.0, considering a 95\% confidence interval. In 2008 in Brazil there were 321 Physical Education Professionals in primary care and 139 in 2020, representing a decrease of 56\% for the number of professionals linked to Primary Health Care. There was a growth in the density of this professional in most States at first and then a decrease. The State of Espirito Santo stands out, with the highest density of professionals in 2008 and Pernambuco wich in 2012 reached the highest peak among the States. The temporal trend varied between Brazilian states, Goiás was the only one that showed an increase in the period from 2008 to 2020, while in the Southeast region all states showed a decrease. Encouraging the practice of supervised and guided physical activity is important to help increase the workforce of Physical Education Professionals in Primary Health Care, as well as to increase the population's level of physical activity, contributing to acquire health benefits from regular practice of physical activity.

Keywords: Physical activity; Unified Health System; Epidemiology; physical education; workforce.

\section{Introdução}

Globalmente, apesar das evidências que mostram que a promoção da atividade física apresenta crescimento, uma quantidade significativa da população não atinge os níveis recomendados de prática de atividade física por semana ${ }^{1}$, sendo que a recomendação para adultos é de 150 a 300 minutos de atividade física de intensidade moderada por semana para atingir os benefícios à saú$\mathrm{de}^{2}$. É possível identificar associações entre um estilo de vida fisicamente inativo e o aumento de possibilidades de surgimento de diversas doenças ${ }^{3}$.

O aumento da prática de atividade física regular é 
uma estratégia de política pública nacional, e para que isto ocorra o Ministério da Saúde inclui a atividade física como parte do plano de ações estratégicas para o enfrentamento das doenças e agravos não transmissíveis no Brasil de 2021 à 2030, em que uma das metas é aumentar em $30 \%$ a prevalência de prática de atividade física no tempo livre da população e também inclui no eixo de promoção à saúde a estimulação ao desenvolvimento de ambientes saudáveis no trabalho, na escola, na comunidade e nos serviços de saúde no Sistema Único de Saúde (SUS) pela oferta de serviços voltados à prática de atividade física e do lazer ${ }^{4}$.

Cabe ao SUS promover ações de práticas de atividades físicas pela equipe de profissionais nos três níveis de atenção à saúde em destaque na Atenção Primária à Saúde (APS) ${ }^{5}$. A APS é caracterizada por realizar um conjunto de ações individuais e coletivas, sendo realizadas por equipes multiprofissionais, com objetivos de promoção à saúde e prevenção de doenças e agravos, sendo considerado o primeiro local de acesso da população ao sistema de saúde 5 . Um dos profissionais que compõem esta equipe multiprofissional é o Profissional de Educação Física (PEF).

De acordo com o artigo 3o da lei 9696/98 é competência do PEF “coordenar, planejar, programar, supervisionar, dinamizar, dirigir, organizar, avaliar e executar trabalhos, programas, planos e projetos, bem como prestar serviços de auditoria, consultoria e assessoria, realizar treinamentos especializados, participar de equipe multidisciplinar e interdisciplinar e elaborar informes técnicos, científicos e pedagógicos, todos nas áreas de atividades físicas e do desporto" 6 . A prática de atividade física se constitui como uma importante ferramenta não farmacológica para a prevenção das principais condições de saúde que acometem a população ${ }^{7}$.

O planejamento para a força de trabalho suficiente necessita de uma abordagem que seja integrada e flexível, combinando habilidade e produtividade ${ }^{8}$. O recrutamento da força de trabalho em saúde deve atentar-se à região, distritos e instalações, com o mínimo de profissionais necessários para garantir a assistência aos usuários dos serviços de saúde 9 .

Silva ${ }^{10}$ identificou que no período 2013 a 2017 ocorreu um aumento de 140,8\% de PEF com vínculo no SUS, cabe salientar que no estudo citado a coleta de dados e análises foram realizadas com números absolutos de PEF e sem a organização por nível de assistência à saúde, em especial na APS, aumentando a importância de conduzir estudos que identifiquem os locais em que estes profissionais estão inseridos no SUS e que realizem a padronização por carga horária disponível ao acesso do usuário.

Neste sentido, para o conhecimento acerca da densidade profissional na APS no país, estão disponíveis dados somente de outros profissionais de saúde para 10.000 habitantes, no período de 2007 a 2020 ocorreu aumento em $268,1 \%$ para psicólogos, $424,8 \%$ para fisioterapeutas, $297,1 \%$ para fonoaudiólogos, já para terapeutas ocupacionais o aumento de 504,5\% de 2009 a $2020^{11}$.

Entende-se que o PEF é fundamental na promoção da prática de atividade física, contribuindo para o aumento do nível de atividade física da população e reduzindo as chances de desenvolvimento de doenças provenientes da inatividade física, principalmente na APS. Com isso, é importante identificar a força de trabalho do PEF nos serviços de saúde do Brasil, a fim de identificar como encontra-se a sua distribuição. Assim, o objetivo deste trabalho foi analisar a distribuição espaço temporal de Profissionais de Educação Física na APS do SUS nos Estados brasileiros.

\section{Métodos}

Este estudo é de característica ecológica de série temporal sobre a oferta de PEF que ofertam atendimento na APS no SUS.

Os dados utilizados são provenientes do Cadastro Nacional de Estabelecimentos de Saúde, sistema oficial utilizado para cadastrar as informações de todos os estabelecimentos de saúde do país. Este é o registro oficial do Ministério da Saúde referente à capacidade e força de trabalho dos serviços de saúde no Brasil. Os dados estão disponíveis no site do Departamento de Informática do Sistema Único de Saúde (http://cnes. datasus.gov.br). A extração e o pré-processamento dos dados foram realizados no programa $R$ Studio 1.2 e no pacote microdatasus ${ }^{12}$, do banco de dados CNES-PF.

A Classificação Brasileira de Ocupações dos PEF considerados neste estudo foram: Avaliador Físico (224105), Preparador Físico (224120), Profissional de Educação Física na Saúde (224140), Professor de Educação Física do Ensino Fundamental (231315), Professor de Educação Física no Ensino Médio (232120), Professor de Educação Física no Ensino Superior (224410).

As unidades de APS consideradas para este estudo foram: Posto de Saúde; Centro de Saúde/Unidade Básica; Unidade mista; Unidade Móvel Terrestre; 
Unidade Móvel Fluvial; Centro de Apoio à Saúde da Família; Unidade da Academia da Saúde; Serviço de Assistência Domiciliar Isolado; Unidade de cuidado residencial ${ }^{13}$. Os dados foram extraídos inicialmente considerando os municípios brasileiros, e posteriormente agregados por Estados e regiões geográficas.

O período de análise foi de 2008 até 2020, tendo como referência o mês de agosto para cada ano analisado. É recomendado que seja realizada a análise de pelo menos um período de 10 anos da força de trabalho para observar ações de longo prazo, visando o alcance de resultados sustentáveis no desenvolvimento da força de trabalho em saúde ${ }^{14}$. Os dados foram analisados considerando os PEF e as populações específicas de cada Estado do Brasil.

Para corrigir possíveis vieses, optou-se em realizar as análises padronizadas para uma semana de trabalho de 40 horas, visto que há possibilidade de diferentes jornadas de trabalho dos PEF, impactando nas atuações nos serviços de saúde.

A densidade de trabalhadores de PEF, em nível estadual e por ano, foi calculada a partir da soma do número de horas trabalhadas dividido por 40 horas semanais ${ }^{15}$. O indicador foi então calculado pela densidade de trabalhadores de PEF pela população em cada Estado por ano, multiplicado por 10.000 habitantes. Os dados anuais sobre a estimativa da população brasileira de 2008 a 2020 foram obtidos do Instituto Brasileiro de Geografia e Estatística ${ }^{16}$.

A visualização espacial da tendência da densidade de PEF que atuam na APS foi representada por gráficos de linha e mapas, segundo os Estados e agrupados nas regiões geográficas do Brasil para os anos de 2008 a 2020.

Para a análise espacial foram produzidos mapas temáticos utilizando-se o software GeoDa 1.14.0.24, e a base cartográfica do Brasil por Estados foi obtida no sítio eletrônico do Instituto Brasileiro de Geografia e Estatística.

Foram analisadas as tendências temporais da densidade de PEF por ano no Brasil e Estados, para isto foi realizada a análise de regressão no software Joinpoint Regression Program, versão 4.7.0.0, na qual foi estimado o coeficiente de variação percentual média anual, considerando o respectivo intervalo de confiança de $95 \%$. O modelo mais ajustado foi selecionado, no qual a $A n-$ nual Percent Change (APC) foi baseada na tendência de cada segmento, estimando se esses valores eram estatisticamente significativos $(p<0,05)$. Para quantificar a tendência dos anos analisados, foi calculada a Average
Percent Change (AAPC). O AAPC é calculado com base na média geométrica acumulada das tendências de APC, com pesos iguais para os comprimentos de cada segmento durante o intervalo fixo. Os testes de significância utilizados baseiam-se no método de permutação de Monte Carlo e no cálculo da variação percentual anual da razão, utilizando o logaritmo da razão ${ }^{17,18}$.

Os dados utilizados nesta pesquisa são de domínio público e estão disponíveis no site do Ministério da Saúde e do Instituto Brasileiro de Geografia e Estatística. Segundo Resolução CONEP/CNS no 510/2016, pesquisas com base em dados secundário de domínio público são dispensadas de apreciação pelo comitê de ética em pesquisa.

\section{Resultados}

No Brasil, em 2008 haviam 321 PEF cadastrados na APS e 139 em 2020, o que representa uma diminuição de $56 \%$ na quantidade deste profissional. Para a densidade de $\mathrm{PEF}$, em primeiro momento, ocorreu crescimento para a maioria dos Estados, até aproximadamente o ano de 2012, após este período os Estados apresentam decréscimo dessa densidade. O Estado do Espírito Santo, apresenta diferencial em relação aos outros, visto que a densidade de PEF já inicia em 2008 com dados superiores aos demais. Pernambuco atingiu em 2012 a maior densidade entre os Estados. Rondônia foi o Estado em que é quase imperceptível o aumento ou a diminuição ao longo dos anos (Figura 1).

É possível identificar a variação da força de trabalho de PEF na APS nos Estados a partir da visualização espacial no mapa (Figura 2). Em 2012 ocorreu uma mudança importante na distribuição deste profissional, visto que a partir deste momento observa-se a diminuição de PEF nos Estados do Brasil

Observando a tendência temporal, de 2008 a 2020 apenas o Estado de Goiás apresentou crescimento significativo (AAPC: 16,2), já o Espírito Santo apresentou o maior decréscimo significativo (AAPC: -42,9). Quando observado por região, o Sudeste apresentou o pior resultado, com decréscimo em todos os seus Estados (Tabela 1).

\section{Discussão}

Os resultados mostraram que ocorre um padrão na densidade e tendência temporal de PEF na APS, em que a maioria dos Estados aumenta de 2008 a 2012, seguido de um decréscimo até 2020.

Entre 2008 a 2013 houve crescimento de 145\% 


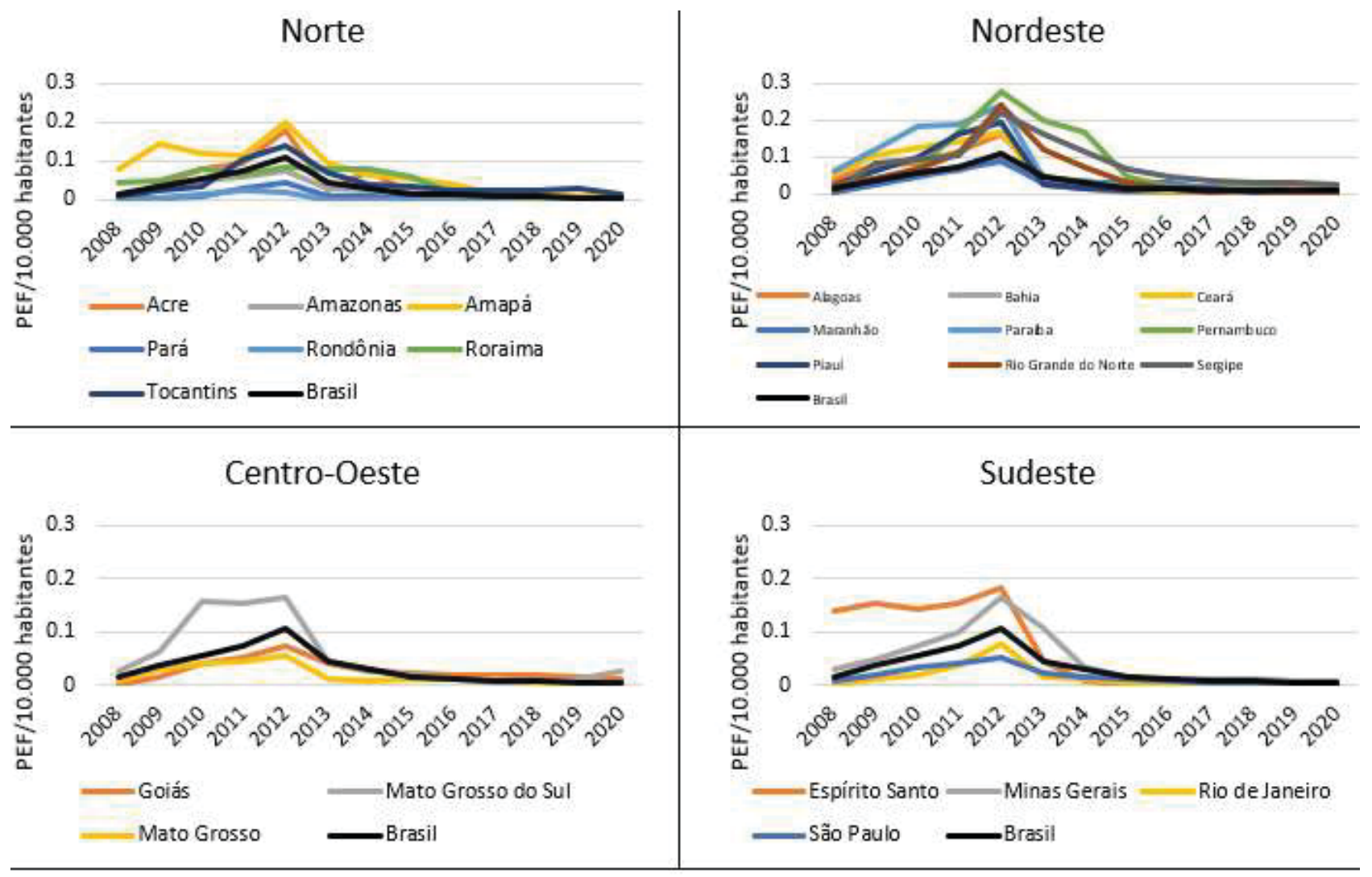

Sul

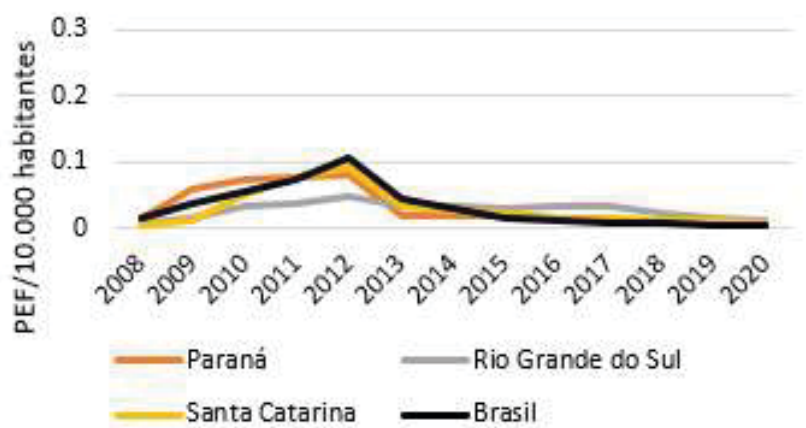

Figura 1 - Densidade* de Profissionais de Educação Física na Atenção Primária à Saúde, segundo os Estados. Brasil, 2008 - 2020. *Densidade: 40 horas de Profissional de Educação Física por 10.000 habitantes.

dos PEF nos estabelecimentos de Centro de Saúde/ Unidade Básica de Saúde devidamente cadastrados no Cadastro Nacional de Estabelecimentos de Saúde ${ }^{5}$, período que corrobora com a densidade de PEF no Brasil de nosso estudo, aumento de $181 \%$, porém, quando observado de 2013 a 2020 ocorreu a diminuição em 84\%, retornando a densidade abaixo do início da análise, em que, $2008=321$ e $2020=139$ PEF.

Em 2017 o Estado do Piauí possuía a relação mais favorável para habitantes/PEF de 16.341 e a menos favorável foi o Distrito Federal com 303.944 habitantes/ PEF para profissionais no SUS ${ }^{10}$. Em nosso estudo, para o ano de 2017 o Estado com a maior densidade de PEF na APS foi Sergipe com 0.0034 e o menor o Rio de Janeiro com 0.0002, além de Distrito Federal, Espírito Santo e Rondônia com a densidade igual a 0.

O Programa Academia da Saúde e o Núcleo de Apoio à Saúde da Família podem ter contribuído para o aumento da taxa deste profissional. As Academias da Saúde foram criadas e consideradas um importante programa, visando a continuidade das ações realizadas na APS, com trabalhos de promoção da saúde e prevenção de doenças, em que as ações são realizadas por equipe multiprofissional, considerando os mesmos profissio- 

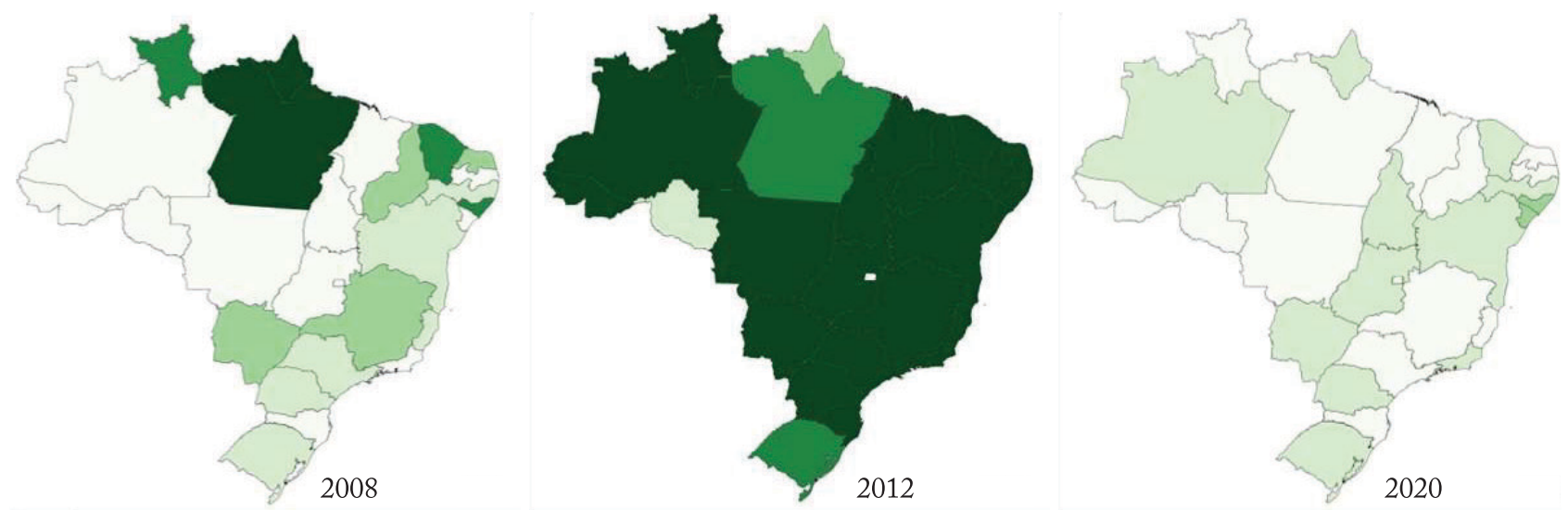

Densidade de profissionais de Educação Física por estado na Atenção Primária à Saúde

$\square 0$
$\square 0,01-0,02$
$\square, 02-0,03$
$0,03-0,04$
$0,04-0,05$
Acima de 0,05

Figura 2 - Visualização espacial da força de trabalho dos Profissionais de Educação Física na Atenção Primária à Saúde segundo os Estados por 10.000 habitantes. Brasil, 2008, 2012 e 2020.

nais atuantes do Núcleo de Apoio à Saúde da Famíliaa ${ }^{19}$.

Em 2017, na publicação da Política Nacional de Atenção Básica ${ }^{20}$ e posteriormente em 2019 com o Programa Previne Brasil21 , é possível observar o movimento de mudança na política nacional em relação ao Núcleo de Apoio à Saúde da Família, com a proposta de que as equipes multiprofissionais passassem a complementar as equipes de Saúde da Família e as equipes tradicionais da APS. Esses acontecimentos podem alterar a disponibilidade de carga horária dos PEF no decorrer dos anos futuros.

Apesar de não terem ocorrido na mesma proporção, todas as categorias profissionais de nível superior em saúde apresentaram crescimento com a criação do SUS a partir de $1988^{5}$. Vale ressaltar que a Profissão de Educação Física neste período ainda não era devidamente regulamentada, o que pode interferir diretamente na inserção deste profissional nos serviços de saúde. A regulamentação ocorreu apenas em 1998, através da lei No 9.696, junto com a criação do Conselho Federal e os Conselhos Regionais de Educação Física ${ }^{6}$.

Para uma boa gestão da força de trabalho em saúde é preciso uma análise do ambiente e da governança e implementação de políticas de força de trabalho em saúde, orientando a identificação dos níveis e intervenções mais necessárias para o momento, entendendo que não é possível identificar as melhores práticas e repli- cá-las de maneira igual para todos os países, as ações precisam ser planejadas e realizadas de acordo com a necessidade de cada local ${ }^{22}$.

Identificar somente a falta do planejamento não é suficiente para compreender as razões do "não planejamento da força de trabalho”, visto a sua importância e necessidade desde o início da construção do SUS ${ }^{23}$.

No Brasil, a cobertura das equipes de saúde da família é maior nos locais em que há menos médicos disponíveis para atendimento por meio dos serviços públicos de saúde, no nordeste estas equipes são responsáveis por cobrir $65 \%$ da população, já no sudeste a cobertura pelas equipes é de $32 \%$ da população ${ }^{23}$. Neste estudo o Nordeste foi o local com maior densidade de PEF.

É necessário que o planejamento de recursos humanos seja preciso e executado no tempo adequado, considerando os possíveis atritos na implementação de políticas no setor da saúde ${ }^{8}$. A quantidade da força de trabalho em saúde em relação a população é um indicador importante para compreender a disponibilidade da força em trabalho em saúde para a população ${ }^{24}$.

O Vigitel identificou que no ano de 2020 apenas $36,8 \%$ dos adultos brasileiros atingiam a recomendação de atividade física de lazer de 150 minutos por semana de intensidade moderada ${ }^{25}$. É de conhecimento geral que os bons níveis de atividade física auxiliam na prevenção e tratamento de algumas doenças, assim, é 
Tabela 1 - Tendência temporal da força de trabalho dos Profissionais de Educação Física na Atenção Primária à Saúde segundo os Estados por 10.000 habitantes. Brasil, 2008 - 2020.

\begin{tabular}{|c|c|c|c|c|c|c|}
\hline Reg. & Estado & Seg. & $\begin{array}{c}\text { Ano } \\
\text { Inicial } \\
\text { (Dens.) }\end{array}$ & $\begin{array}{c}\text { Ano } \\
\text { Final } \\
\text { (Dens.) }\end{array}$ & $\begin{array}{c}\text { APC } \\
\text { IC 95\% }\end{array}$ & $\begin{array}{l}\text { AAPC } \\
\text { IC 95\% }\end{array}$ \\
\hline \multirow{21}{*}{ 䒕 } & \multirow{3}{*}{ Acre } & 1 & $\begin{array}{c}2009 \\
(0,03)\end{array}$ & $\begin{array}{l}2017 \\
(0,01)\end{array}$ & $-20,9^{*}$ & $-20,9^{*}$ \\
\hline & & 2 & - & - & - & - \\
\hline & & 3 & - & - & - & - \\
\hline & \multirow{3}{*}{ Amapá } & 1 & $\begin{array}{l}2008 \\
(0,08)\end{array}$ & $\begin{array}{c}2012 \\
(0,20)\end{array}$ & 11,1 & \multirow{2}{*}{$-19,2^{*}$} \\
\hline & & 2 & $\begin{array}{c}2012 \\
(0,20)\end{array}$ & $\begin{array}{c}2020 \\
(0,01)\end{array}$ & $-31,1^{*}$ & \\
\hline & & 3 & - & - & - & - \\
\hline & \multirow{3}{*}{ Amazonas } & 1 & $\begin{array}{c}2008 \\
(<0,01)\end{array}$ & $\begin{array}{c}2011 \\
(0,06)\end{array}$ & $96,3^{*}$ & \multirow{2}{*}{$-6,4$} \\
\hline & & 2 & $\begin{array}{c}2011 \\
(0,06)\end{array}$ & $\begin{array}{c}2017 \\
(<0,01)\end{array}$ & $-35,3^{*}$ & \\
\hline & & 3 & - & - & - & - \\
\hline & \multirow{3}{*}{ Pará } & 1 & $\begin{array}{c}2008 \\
(<0,01)\end{array}$ & $\begin{array}{c}2011 \\
(0,03)\end{array}$ & $112,0^{*}$ & \multirow[b]{2}{*}{$-5,5$} \\
\hline & & 2 & $\begin{array}{c}2011 \\
(0,03)\end{array}$ & $\begin{array}{c}2020 \\
(<0,01)\end{array}$ & $-27,8^{*}$ & \\
\hline & & 3 & - & - & - & - \\
\hline & \multirow{3}{*}{ Rondônia } & 1 & $\begin{array}{c}2009 \\
(<0,01)\end{array}$ & $\begin{array}{c}2012 \\
(0,01)\end{array}$ & 46,4 & 46,4 \\
\hline & & 2 & - & - & - & - \\
\hline & & 3 & - & - & - & - \\
\hline & \multirow{3}{*}{ Roraima } & 1 & $\begin{array}{l}2008 \\
(0,04)\end{array}$ & $\begin{array}{l}2014 \\
(0,08)\end{array}$ & 11,1 & \multirow{2}{*}{$-12,9$} \\
\hline & & 2 & $\begin{array}{c}2014 \\
(0,08)\end{array}$ & $\begin{array}{c}2017 \\
(0,01)\end{array}$ & $-46,4^{*}$ & \\
\hline & & 3 & - & - & - & - \\
\hline & \multirow{3}{*}{ Tocantins } & 1 & $\begin{array}{c}2008 \\
(<0,01)\end{array}$ & $\begin{array}{c}2011 \\
(0,10)\end{array}$ & $132,4^{*}$ & \multirow{2}{*}{4,2} \\
\hline & & 2 & $\begin{array}{c}2011 \\
(0,10)\end{array}$ & $\begin{array}{c}2020 \\
(0,01)\end{array}$ & $-20,3^{*}$ & \\
\hline & & 3 & - & - & - & - \\
\hline \multirow{9}{*}{ 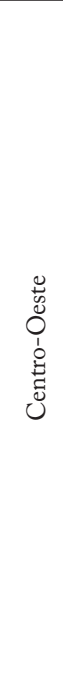 } & \multirow{3}{*}{ Goiás } & 1 & $\begin{array}{c}2008 \\
(<0,01)\end{array}$ & $\begin{array}{l}2010 \\
(0,04)\end{array}$ & $474,6^{*}$ & \multirow{2}{*}{$16,2^{*}$} \\
\hline & & 2 & $\begin{array}{c}2010 \\
(0,04)\end{array}$ & $\begin{array}{c}2020 \\
(0,01)\end{array}$ & $-15,6^{*}$ & \\
\hline & & 3 & - & - & - & - \\
\hline & \multirow{3}{*}{ Mato Grosso } & 1 & $\begin{array}{c}2008 \\
(<0,01)\end{array}$ & $\begin{array}{c}2010 \\
(0,04)\end{array}$ & 108,1 & \multirow{2}{*}{$-5,9$} \\
\hline & & 2 & $\begin{array}{c}2010 \\
(0,04)\end{array}$ & $\begin{array}{c}2020 \\
(<0,01)\end{array}$ & $-19,7^{*}$ & \\
\hline & & 3 & - & - & - & - \\
\hline & \multirow{3}{*}{$\begin{array}{l}\text { Mato Grosso } \\
\text { do Sul }\end{array}$} & 1 & $\begin{array}{l}2008 \\
(0,02)\end{array}$ & 2011 & $85,5^{*}$ & \multirow{3}{*}{$-14,0$} \\
\hline & & 2 & $\begin{array}{c}2011 \\
(0,15)\end{array}$ & 2018 & $-44,5^{*}$ & \\
\hline & & 3 & $\begin{array}{c}2018 \\
(<0,01)\end{array}$ & $\begin{array}{c}2020 \\
(0,02)\end{array}$ & $193,2^{*}$ & \\
\hline
\end{tabular}

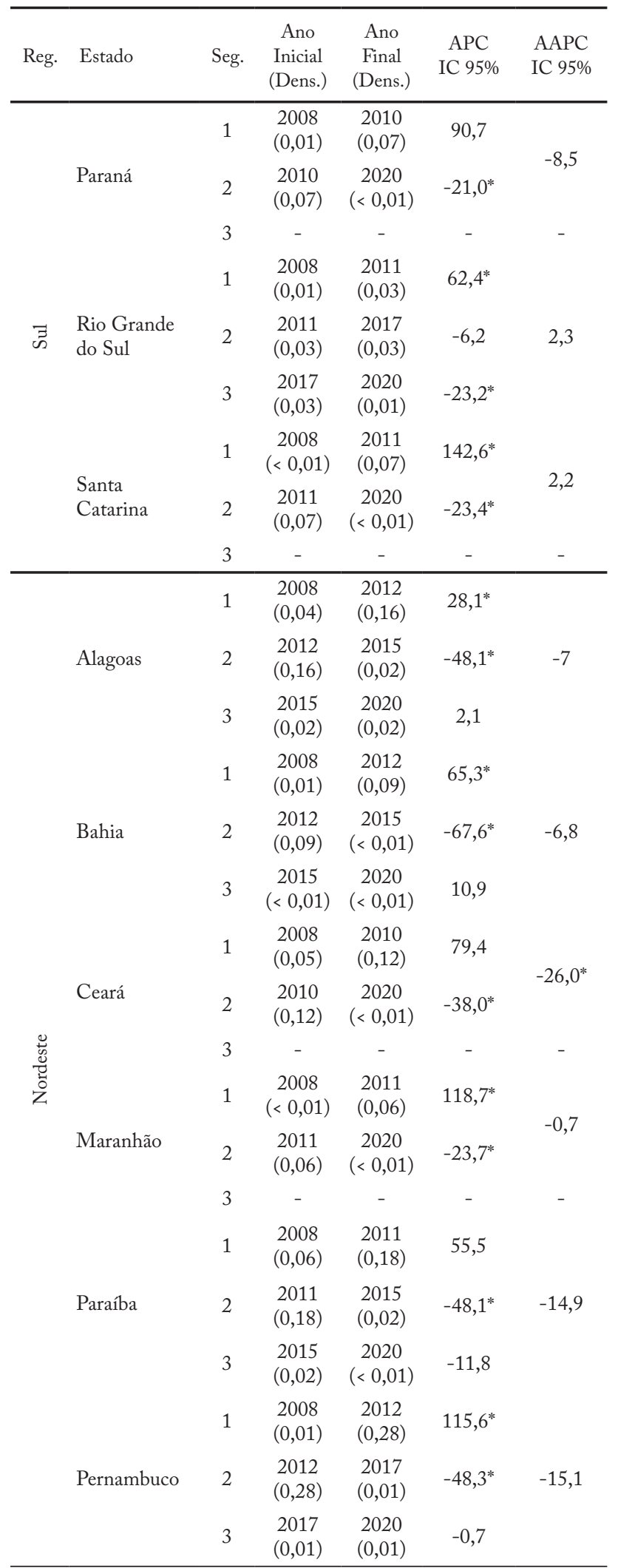

Continua... 
Continuação da Tabela 1 - Tendência temporal da força de trabalho dos Profissionais de Educação Física na Atenção Primária à Saúde segundo os Estados por 10.000 habitantes. Brasil, 2008 - 2020.

\begin{tabular}{|c|c|c|c|c|c|c|}
\hline Reg. & Estado & Seg. & $\begin{array}{c}\text { Ano } \\
\text { Inicial } \\
\text { (Dens.) }\end{array}$ & $\begin{array}{c}\text { Ano } \\
\text { Final } \\
\text { (Dens.) }\end{array}$ & $\begin{array}{c}\text { APC } \\
\text { IC 95\% }\end{array}$ & $\begin{array}{l}\text { AAPC } \\
\text { IC } 95 \%\end{array}$ \\
\hline \multirow{9}{*}{ 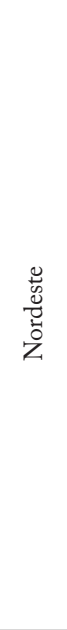 } & \multirow{3}{*}{ Piauí } & 1 & $\begin{array}{l}2008 \\
(0,02)\end{array}$ & $\begin{array}{l}2011 \\
(0,16)\end{array}$ & $108,9^{*}$ & \multirow{3}{*}{$-13,6$} \\
\hline & & 2 & $\begin{array}{c}2011 \\
(0,16)\end{array}$ & $\begin{array}{c}2014 \\
(0,01)\end{array}$ & $-58,2$ & \\
\hline & & 3 & $\begin{array}{l}2014 \\
(0,01)\end{array}$ & $\begin{array}{c}2019 \\
(<0,01)\end{array}$ & $-21,4$ & \\
\hline & \multirow{3}{*}{$\begin{array}{l}\text { Rio Grande } \\
\text { do Norte }\end{array}$} & 1 & $\begin{array}{l}2008 \\
(0,02)\end{array}$ & $\begin{array}{c}2012 \\
(0,24)\end{array}$ & $77,7^{*}$ & \multirow{2}{*}{$-15,0^{*}$} \\
\hline & & 2 & $\begin{array}{l}2012 \\
(0,24)\end{array}$ & $\begin{array}{c}2018 \\
(<0,01)\end{array}$ & $-48,0^{*}$ & \\
\hline & & 3 & - & - & - & - \\
\hline & \multirow{3}{*}{ Sergipe } & 1 & $\begin{array}{c}2008 \\
(<0,01)\end{array}$ & $\begin{array}{l}2010 \\
(0,09)\end{array}$ & $458,7^{*}$ & \multirow{2}{*}{11,5} \\
\hline & & 2 & $\begin{array}{c}2010 \\
(0,09)\end{array}$ & $\begin{array}{l}2020 \\
(0,02)\end{array}$ & $-19,2^{*}$ & \\
\hline & & 3 & - & - & - & - \\
\hline \multirow{12}{*}{ 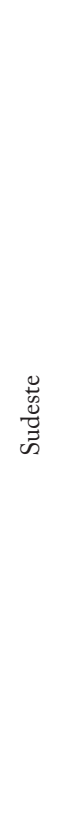 } & \multirow{3}{*}{$\begin{array}{l}\text { Espírito } \\
\text { Santo }\end{array}$} & 1 & $\begin{array}{l}2008 \\
(0,14)\end{array}$ & $\begin{array}{l}2012 \\
(0,18)\end{array}$ & $-4,3$ & \multirow[b]{2}{*}{$42,9^{*}$} \\
\hline & & 2 & $\begin{array}{c}2012 \\
(0,18)\end{array}$ & $\begin{array}{c}2016 \\
(<0,01)\end{array}$ & $-65,9^{*}$ & \\
\hline & & 3 & - & - & - & - \\
\hline & \multirow{3}{*}{ Minas Gerais } & 1 & $\begin{array}{l}2008 \\
(0,02)\end{array}$ & $\begin{array}{c}2012 \\
(0,16)\end{array}$ & $58,4^{*}$ & \multirow{3}{*}{$10,2^{*}$} \\
\hline & & 2 & $\begin{array}{l}2012 \\
(0,16)\end{array}$ & $\begin{array}{l}2015 \\
(0,01)\end{array}$ & $-57,0^{*}$ & \\
\hline & & 3 & $\begin{array}{l}2015 \\
(0,01)\end{array}$ & $\begin{array}{c}2020 \\
(<0,01)\end{array}$ & $-11,2^{*}$ & \\
\hline & \multirow{3}{*}{$\begin{array}{l}\text { Rio de } \\
\text { Janeiro }\end{array}$} & 1 & $\begin{array}{c}2008 \\
(<0,01)\end{array}$ & $\begin{array}{c}2011 \\
(0,03)\end{array}$ & $187,8^{*}$ & \multirow[b]{2}{*}{$-7,1$} \\
\hline & & 2 & $\begin{array}{l}2011 \\
(0,03)\end{array}$ & $\begin{array}{c}2020 \\
(<0,01)\end{array}$ & $-36,2^{*}$ & \\
\hline & & 3 & - & - & - & - \\
\hline & \multirow{3}{*}{ São Paulo } & 1 & $\begin{array}{c}2008 \\
(<0,01)\end{array}$ & $\begin{array}{c}2011 \\
(0,04)\end{array}$ & $75,8^{*}$ & \multirow{2}{*}{$-11,1^{*}$} \\
\hline & & 2 & $\begin{array}{l}2011 \\
(0,04)\end{array}$ & $\begin{array}{c}2020 \\
(<0,01)\end{array}$ & $-29,1^{*}$ & \\
\hline & & 3 & - & - & - & - \\
\hline
\end{tabular}

Seg. $=$ Segmento; Ano Inicial: Ano inicial do segmento; Ano Final = Ano final do segmento; Dens. = densidade profissional por 10.000 habitantes; $\mathrm{APC}=$ Annual Percent Change; $\mathrm{AAPC}=$ Average Annual Percent Change; IC95\% = Intervalo de confiança de 95\%. *Estatisticamente significativo no nível de 5\%.

importante trabalhar no incentivo a criação de políticas públicas de promoção da saúde por meio da prática de atividade física. Aumentar o nível de atividade física da população faz parte do plano de ações estratégicas para o enfrentamento das doenças crônicas e agravos não transmissíveis no Brasil nos anos de 2021 a $2030^{4}$.

Atuar em fatores de risco pode evitar cerca de $80 \%$ das doenças cardiovasculares e diabetes tipo II, dentre estes, destaca-se os modificáveis, incluindo a inatividade física. Com isso, estima-se que o sedentarismo eleva os custos em saúde em aproximadamente US\$ 54 bilhões por ano, assim, mudanças no padrão de atividade física da população são necessárias para combater parte das doenças crônicas não transmissíveis ${ }^{26}$

A inatividade física possui um papel importante no aumento dos gastos com saúde pública, dessa forma, pode-se justificar o desenvolvimento de estratégias de prevenção de doenças crônicas e consequentemente reduzir os custos em excesso com procedimentos de saúde, através da promoção da atividade física para a população ${ }^{27}$. Não aumentar os níveis de atividade física da população, poderá levar ao aumento dos custos, causando uma consequência negativa aos serviços de saúde, ambiente, desenvolvimento econômico, qualidade de vida e no bem-estar de maneira geral ${ }^{28}$.

Os dados mais recentes do Vigitel demonstram que a cidade de Florianópolis (11\%) possui a menor frequência de indivíduos fisicamente inativos e Rio Branco $(20,9 \%)$ a maior, e considerando todo o Brasil está frequência é de $14,9 \%{ }^{25}$. Intervenções de atividade física na APS seriam importantes para garantir um maior acesso da população em ações de promoção da saúde, pelo menos 120 minutos semanais já são suficientes em melhorar a qualidade de vida, porém, nem sempre é possível devido à baixa disponibilidade do $\mathrm{PEF}$ para orientação da população ${ }^{29}$. No Brasil, a cada dez unidades de saúde quatro relatam ter intervenção com atividade física, geralmente grupos de caminhada ${ }^{30}$.

Em 2021, ocorreu o lançamento do Guia de Atividade Física para a População Brasileira do Ministério da Saúde, com as primeiras informações e recomendações de atividade física, afim de melhorar os níveis de atividade física da população, influenciando a qualidade de vida e promovendo saúde por meio de um estilo de vida ativo ${ }^{31}$. O PEF é responsável por realizar a orientação adequada da prática de atividade física, a fim de obter benefícios para a saúde ${ }^{10}$. Destaca-se então, a necessidade da prática regular da atividade física, principalmente com a orientação e supervisão adequada do PEF na APS para melhores resultados.

Algumas limitações devem ser consideradas neste estudo. Os dados secundários podem apresentar possíveis problemas de registro, porém, são os dados oficiais do Ministério da Saúde do Brasil ${ }^{32}$. Foi utilizada a população estimada para a análise, baseada no censo de 2010, em razão da ausência de censo anual. 
Havia pouca disponibilidade de PEF na APS, a priori ocorreu aumento da força de trabalho, até aproximadamente 2012, após este período observa-se diminuição até 2020, este padrão é observado para a maioria dos Estados do Brasil. Embora há incentivo de prática de atividade física para a promoção da saúde e prevenção de algumas doenças, visando tornar a população fisicamente mais ativa para benefícios de sua saúde, há pouco estímulo para que a prática de atividade física seja realizada sob supervisão e orientação do PEF. A baixa densidade de PEF inseridos na APS pode implicar na realização das práticas corporais de qualidade que as ações e os programas de promoção da saúde incentivam, com isso a criação de políticas públicas que recomendam a realização de atividade física com supervisão e orientação do PEF são necessárias.

\section{Conflito de interesse}

Os autores declaram não haver conflito de interesse.

\section{Financiamento}

O presente trabalho foi realizado com apoio da Coordenação de Aperfeiçoamento de Pessoal de Nível Superior - Brasil (CAPES) - Código de Financiamento 001.

\section{Contribuição dos autores}

Todos os autores participaram ativamente do estudo. Silva DB, contribuiu com a análise e interpretação dos dados e foi uma das principais contribuintes na redação do manuscrito. Sixel TRS, contribuiu com as análises e interpretação dos dados, e revisão do manuscrito. Medeiros AA, contribuiu com as análises e interpretação dos dados, e revisão do manuscrito. Schmitt ACB, contribuiu com a concepção do estudo, análise e interpretação dos dados, e com a redação do manuscrito.

\section{Referências}

1. Guthold R, Stevens GA, Riley LM, et al. Worldwide trends in insufficient physical activity from 2001 to 2016: a pooled analysis of 358 population-based surveys with 1.9 million participants. The Lancet Global Health. 2018;6:e1077-e1086.

2. World Health Organization. WHO guidelines on physical activity and sedentary behaviour: at a glance. Geneva, 2020.

3. Ding D, Lawson KD, Kolbe-AlexanderTL, et al. The economic burden of physical inactivity: a global analysis of major noncommunicable diseases. Lancet. 2016;388: 1311-24.

4. Ministério da Saúde. Plano de ações estratégicas para o enfrentamento das doenças crônicas e agravos não transmissíveis no Brasil. Brasília, DF, 2020.

5. Carvalho MN de, Gil CRR, Costa EMOD, et al. Needs and dynamics of the Primary Healthcare workforce in Brazil. Ciênc saúde colet. 2018;23: 295-302.

6. L9696, http://www.planalto.gov.br/ccivil_03/leis/19696.htm (accessed 27 April 2021).
7. Lima WP. Educação Física e Saúde: perspectivas de atuação profissional. Rev Bras Fisiol Exerc. 2019;18: 64-9.

8. Lopes MA, Almeida ÁS, Almada-Lobo B. Handling healthcare workforce planning with care: where do we stand? Hum. Resour. Health 2015;13:38.

9. Asamani JA, Ismaila H, Plange A, et al. The cost of health workforce gaps and inequitable distribution in the Ghana Health Service: an analysis towards evidence-based health workforce planning and management. Hum. Resour. Health. 2021;19:43.

10. Silva PSC. Physical Education Professionals in the Unified Health System: an analysis of the brazilian registry of health institutions between 2013 and 2017. Rev Bras Ativ Fís Saúde. 2019;23:1-8.

11. da Silva DB, dos Santos Sixel TR, de Almeida Medeiros A, et al. The workforce for rehabilitation in primary health care in Brazil. Hum. Resour. Health 2021; 19: 127.

12. Saldanha R de F, Bastos RR, Barcellos C. Microdatasus: pacote para download e pré-processamento de microdados do Departamento de Informática do SUS (DATASUS). Cad Saúde Pública; 35. Epub ahead of print 16 September 2019.

13. Brasil M da S. Tipo de estabelecimento. DATASUS, http:// tabnet.datasus.gov.br/cgi/cnes/tipo_estabelecimento.htm (2020, accessed 10 November 2020).

14. World Health Organization. A Universal Truth: no health without a workforce. Geneva: Global Health Workforce Alliance and Word Health Organization, 2013.

15. UFMG (ed). Construção do Índice de escassez de profissionais de saúde, http://epsm.nescon.medicina.ufmg. br/epsm/Relate_Pesquisa/Index_relatorio.pdf (2010).

16. World Health Organization. National Health Workforce Accounts: A Handbook. Disponível em:url:https://apps.who. int/iris/bitstream/handle/10665/259360/9789241513111eng.pdf (citado 2017).

17. Kim HJ, Fay MP, Feuer EJ, et al. Permutation tests for joinpoint regression with applications to cancer rates. Stat Med. 2000;19:335-51.

18. Kim H-J, Fay MP, Yu B, et al. Comparability of segmented line regression models. Biometrics. 2004;60:1005-1014.

19. Brasil M da S. Portaria No 1.402, http://bvsms.saude.gov.br/ bvs/saudelegis/gm/2011/prt1402_15_06_2011.html (2011, accessed 10 November 2020).

20. Brasil M da S. Portaria No 2.436, https://bvsms.saude.gov. br/bvs/saudelegis/gm/2017/prt2436_22_09_2017.html (2017, accessed 10 November 2020).

21. Diário Oficial da União. Portaria No 2.979. 2019; 3.

22. Cometto G, Buchan J, Dussault G. Developing the health workforce for universal health coverage. Bull World Health Organ. 2020; 98:109-116.

23. Carvalho $M$ de, Santos NR dos, Campos GW de S. A construção do SUS e o planejamento da força de trabalho em saúde no Brasil: breve trajetória histórica. Saúde debate. 2013;37:372-87.

24. Karan A, Negandhi H, Hussain S, et al. Size, composition and distribution of health workforce in India: why, and where to invest? Hum. Resour. Health. 2021;19:39.

25. Brasil. Ministério da Saúde. Secretaria de Vigilância em Saúde. Departamento de Análise em Saúde e Vigilância de Doenças Não Transmissíveis. Vigitel Brasil 2020: vigilância de fatores de risco e proteção para doenças crônicas por inquérito telefônico: estimativas sobre frequência e distribuição sociodemográfica de fatores de risco e proteção para doenças crônicas nas capitais dos 26 estados brasileiros e no Distrito Federal em 2020 / Ministério da Saúde, Secretaria de Vigilância em Saúde, Departamento de Análise em Saúde e Vigilância de Doenças Não Transmissíveis. - Brasília: Ministério da Saúde, 2021.124 p. : il. 
26. Ministério da Saúde. Plano Nacional de Saúde 2020-2023. 2020. Disponível em: https://bvsms.saude.gov.br/bvs/ publicacoes/plano_nacional_saude_2020_2023.pdf.

27. Bueno DR, Marucci M de FN, Codogno JS, et al. Os custos da inatividade física no mundo: estudo de revisão. Ciênc saúde colet. 2016;21:1001-10.

28. Organization WH. Global Action Plan on Physical Activity 2018-2030: More Active People for a Healthier World. World Health Organization, 2019.

29. Silva DB da, Papini CB, Nakamura PM, et al. Efetividade de duas intervenções com diferentes volumes de exercícios físicos na qualidade de vida em mulheres usuárias da Atenção Básica de Saúde. Rev. Bras. Ativ. Fis. Saúde. 2018;23:1-9.
30. Teixeira MB, Casanova A, Oliveira CCM, et al. Evaluation of health promotion practices: a look from the participating teams of the National Program for Access and Quality Improvement in Primary Care. Saúde debate. 2014;38:52-68.

31. Ministério da Saúde. Guia de Atividade Física para a População Brasileira. 2021.

32. Rocha TAH, Silva NC da, Barbosa ACQ, et al. Cadastro Nacional de Estabelecimentos de Saúde: evidências sobre a confiabilidade dos dados. Ciênc saúde colet. 2018;23:229-40.

Recebido: 30/09/2021

Aprovado: 22/12/2021

\section{Como citar este artigo:}

Silva DB, Sixel TRS, Medeiros AA, Schmitt ACB. Força de trabalho de Profissionais de Educação Física na Atenção Primária à Saúde. Rev Bras Ativ Fís Saúde. 2022;27:e0240. DOI: 10.12820/rbafs.27e0240 